\title{
FiSSA at SemEval-2020 Task 9: Fine-tuned For Feelings
}

\author{
Bertelt Braaksma Richard Scholtens Stan van Suijlekom Remy Wang Ahmet Üstün \\ University of Groningen \\ Groningen, the Netherlands \\ \{b.braaksma.1, j.f.p.scholtens, s.van.suijlekom, r.wang.7\}@student.rug.nl, a.ustun@rug.nl
}

\begin{abstract}
In this paper, we present our approach for sentiment classification on Spanish-English codemixed social media data in the SemEval-2020 Task 9. We investigate performance of various pre-trained Transformer models by using different fine-tuning strategies. We explore both monolingual and multilingual models with the standard fine-tuning method. Additionally, we propose a custom model that we fine-tune in two steps: once with a language modeling objective, and once with a task-specific objective. Although two-step fine-tuning improves sentiment classification performance over the base model, the large multilingual XLM-RoBERTa model achieves best weighted F1-score with 0.537 on development data and 0.739 on test data. With this score, our team jupitter placed tenth overall in the competition.
\end{abstract}

\section{Introduction}

Code-mixing is a phenomenon in which two or more languages are used in a single utterance. It occurs at various levels of linguistic structure: across sentences (i.e., inter-sentential), within a sentence (i.e.,intrasentential), or at the word/morpheme level. In addition to spoken language, this phenomenon has become especially prevalent on social media. As monolingual systems cannot deal with the code-mixed data, it poses a major challenge for even the most standard NLP tasks. To this end, SemEval 2020 Task 9 (Patwa et al., 2020) proposes the sentiment analysis task for code-mixed social media text, specifically on English-Spanish (Spanglish) and English-Hindi (Hinglish) language pairs.

In this paper, we present our approach called Fine-tuned Spanglish Sentiment Analysis, or FiSSA for short. We focus on various pre-trained language models for Spanglish sentiment classification by fine-tuning their contextualized word embeddings. By doing so, we examine two challenging aspects of code-mixed language processing: (a) Multilinguality, (b) Domain. We firstly compare monolingual models with their multilingual counterparts to evaluate the multilingual solution on code-switching data, considering the first aspect. Secondly, to see the domain effect, we further fine-tune the multilingual model on domain-specific unlabeled data. Finally, we use the most recent state-of-the-art pre-trained model and compare it to our custom model with domain information.

Our research shows that fine-tuning a pre-trained language model is a good choice compared to the standard BLSTM model when training data is limited. However, on code-mixed data, monolingual pretrained models tend to perform better on different portions of the data depending on the use of languages. As the best alternative, a large multilingual model provides better generalization and results in a stronger performance.

\section{Background}

Code-Mixed Text Processing Only a limited amount of research has been done in the field of sentiment analysis on Spanish-English social media data. However, some writing has been done regarding Spanish-English code-mixing for other NLP tasks, such as part-of-speech tagging (Solorio and Liu, 2008) and language identification (Solorio et al., 2014). In the first shared task of language identification

This work is licensed under a Creative Commons Attribution 4.0 International License.

License details: http://creativecommons.org/licenses/by/4.0/ 
on code-switched data including Spanish-English (SPA-EN), many systems benefited from a combination of machine learning methods such as an SVM, a CRF, an extended Markov Model, and hand crafted or frequency-based features. On SPA-EN, the best performing system employed a CRF classifier by using various character- and word-level features together with external resources that include monolingual corpora with named-entity lists (Bar and Dershowitz, 2014).

With regard to English-Spanish sentiment analysis on social media data, the first English-Spanish code-switching Twitter corpus annotated with sentiment labels was made available in the research conducted by Vilares et al. (2015) and Vilares et al. (2016). In their trinary annotated corpus, a collection of 3062 tweets were annotated by three annotators fluent in both English and Spanish, classifying each tweet as either positive, neutral or negative. They discovered that there was a small advantage to be gained from using a multilingual approach. However, both monolingual and multilingual approaches struggled with code-switching text.

Pre-trained Transformers Deep, Transformer (Vaswani et al., 2017) based language models provide general-purpose contextualized linguistics representations that have shown great success on various NLP tasks (Devlin et al., 2018; Yang et al., 2019; Liu et al., 2019). These models are pre-trained on large unannotated corpora, and then fine-tuned for downstream tasks according to task-specific objectives. As well as monolingual Transformer models, multilingual models that are pre-trained on the concatenation of monolingual corpora from multiple languages, have enabled significant advances in multilingual NLP (Devlin et al., 2018; Lample and Conneau, 2019; Conneau et al., 2019). Pires et al. (2019) showed that multilingual BERT (Devlin et al., 2018) provides a strong cross-lingual generalization, which allows for the incorporation of information from multiple languages, for example in a code-switching scenario.

\section{System overview}

\subsection{Baseline}

As a baseline, we used a standard bidirectional LSTM (Hochreiter and Schmidhuber, 1997) with pretrained word embeddings. In order to combine English and Spanish words in the BLSTM, we use stacked embeddings which were a mix of Flair English and Flair Spanish word embeddings Akbik et al., 2019).

\subsection{Pre-trained Transformer Models}

For our main system, we incorporated different pre-trained language models by fine-tuning them for the sentiment analysis. We picked a selection of monolingual and multilingual models to see how they perform differently on code-switched data. For our monolingual models, we used English BERT-base (Devlin et al., 2018) and Spanish BERT-base (Cañete et al., 2020). They both have the same 'base' architecture, consisting of 12 Transformer blocks with 12 self-attention heads and hidden size of 768 . The English model has a 30k WordPiece (WP) vocabulary (Wu et al., 2016) and the Spanish model has a 31k SentencePiece (SP) vocabulary (Kudo and Richardson, 2018).

For our multilingual models we used multilingual BERT (M-BERT) with a 110k shared WP vocabulary and XLM-RoBERTa (XLM-R) large with a 250k SP vocabulary (Liu et al., 2019). Both models were trained on a concatenation of over 100 languages. However, M-BERT's architecture is the same as that of the monolingual 'base' models, whilst XLM-R has a larger network with 24 Transformer blocks of 16 self-attention heads and hidden size of 1024.

Besides the off-the-shelf models, we also provided a domain-specific custom model by fine-tuning M-BERT with a language modeling objective on the training data. For the task-specific fine-tuning, we applied a softmax classifier over the pooled output the of first token ([CLS]), which gives the sentence representation.

\section{Experimental setup}

\subsection{Data}

We used the training and development datasets provided by the shared-task (Patwa et al., 2020). For comparison, we split the training data into two pieces: $90 \%$ for training and $10 \%$ for development. The 


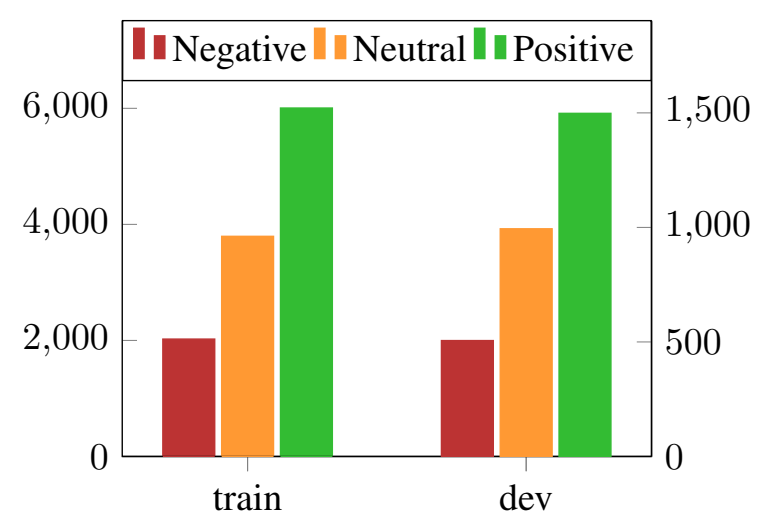

(a) Distribution of sentiment labels in both datasets

\begin{tabular}{lr}
\hline Label & Count \\
\hline lang1 & 7,693 \\
lang2 & 25,435 \\
ambiguous & 43 \\
other & 11,691 \\
mixed & 19 \\
ne & 1,232 \\
unk & 47 \\
fw & 4 \\
\hline
\end{tabular}

(b) Distribution of token-level labels

Figure 1: Details of the training and the development datasets

development data provided by the organizers was then used for the evaluation of all of our models, as the test set was not released. For our final submission, we trained the models by using the whole training set, and used the development data as such.

The three sentiment labels were not equally distributed in the training and development datasets. Positive tweets were over-represented, with roughly 6,000 tweets in training and over 1,400 in development. The second biggest class was neutral with almost 4,000 and 1,000 tweets in training and development respectively. Negative tweets formed the smallest class, with roughly 2,000 tweets in training, and over 500 in development. However, Figure 1a shows that the distribution was similarly skewed in both datasets.

Both datasets were provided with word-level labels including language ids. Figure $1 \mathrm{~b}$ shows the label distribution in the datasets.

\subsection{Implementation}

The baseline system was developed using the Flair library 11 For our LSTM, we used the word embeddings included in the library. In our case, the English and Spanish forward and backward embeddings were used, which were trained on a billion word corpus and Wikipedia respectively. The Flair LSTM classifier was trained using a learning rate of 0.1 and a mini batch size of 32 .

For fine-tuning the pre-trained Transformer models, we used the HuggingFace Transformers ${ }^{2}$ library (Wolf et al., 2019). The existing code had to be slightly modified to add support for sentiment analysis. We fine-tuned all of the models with the exact same hyper-parameters. We set the Adam epsilon to $1^{-8}$ and learning rate to $1^{-5}$, and fine-tuned for 3 epoch 3 .

\section{Results}

The results of each model are presented in Table 1. Precision, recall, and F1-scores are all weighted scores. As the table shows, all of our fine-tuned models performed better than the baseline BLSTM system in every regard, and XLM-RoBERTa large was the best overall, with the highest weighted F1score. Interestingly, when we shift our focus to the monolingual models, we see that the English model performed worse than the Spanish model. The multilingual BERT-model sits right in the middle of these two when we look at the performance metrics.

Our custom model (Custom BERT-base) for which we used a two-step fine-tuning strategy, once with a language modeling objective to inject domain information and once for task-specific fine-tuning, clearly performed better than multilingual BERT. This shows that even very small amount of domain-specific, code-mixed data improves language model quality, when used for further training. However, it still could not match the performance of XLM-Roberta model which is trained on larger corpora and has a larger Transformer network.

\footnotetext{
${ }^{1}$ flairNLP, version 0.4.5, URL: https: / / github.com/flairNLP/flair

2 Transformers, version 2.5.0, URL:https://huggingface.co/transformers/v2.5.0/

${ }^{3}$ The code for our best performing model can be found at https://github.com/barfsma/FiSSA
} 


\begin{tabular}{llcccc}
\hline & Model & Precision & Recall & F1-score & Accuracy \\
\hline 1 & BLSTM & 0.395 & 0.355 & 0.325 & 0.395 \\
2 & English BERT-base & 0.503 & 0.515 & 0.506 & 0.515 \\
3 & Spanish BERT-base & 0.514 & 0.526 & 0.517 & 0.526 \\
4 & Multilingual BERT-base & 0.506 & 0.519 & 0.510 & 0.519 \\
5 & Custom BERT-base & 0.513 & 0.526 & 0.517 & 0.526 \\
6 & XLM-RoBERTa-large & $\mathbf{0 . 5 3 4}$ & $\mathbf{0 . 5 5 1}$ & $\mathbf{0 . 5 3 7}$ & $\mathbf{0 . 5 5 1}$ \\
\hline
\end{tabular}

Table 1: Fine-tuning performance on validation

Figure 2 shows the F1-scores, specified per sentiment label. We can see that all models performed similarly on neutral and positive. However, there is much more variation in the negative class. English BERT for example, had particularly poor performance here. XLM-RoBERTa on the other hand, had no issues and even managed to better its score on neutral.

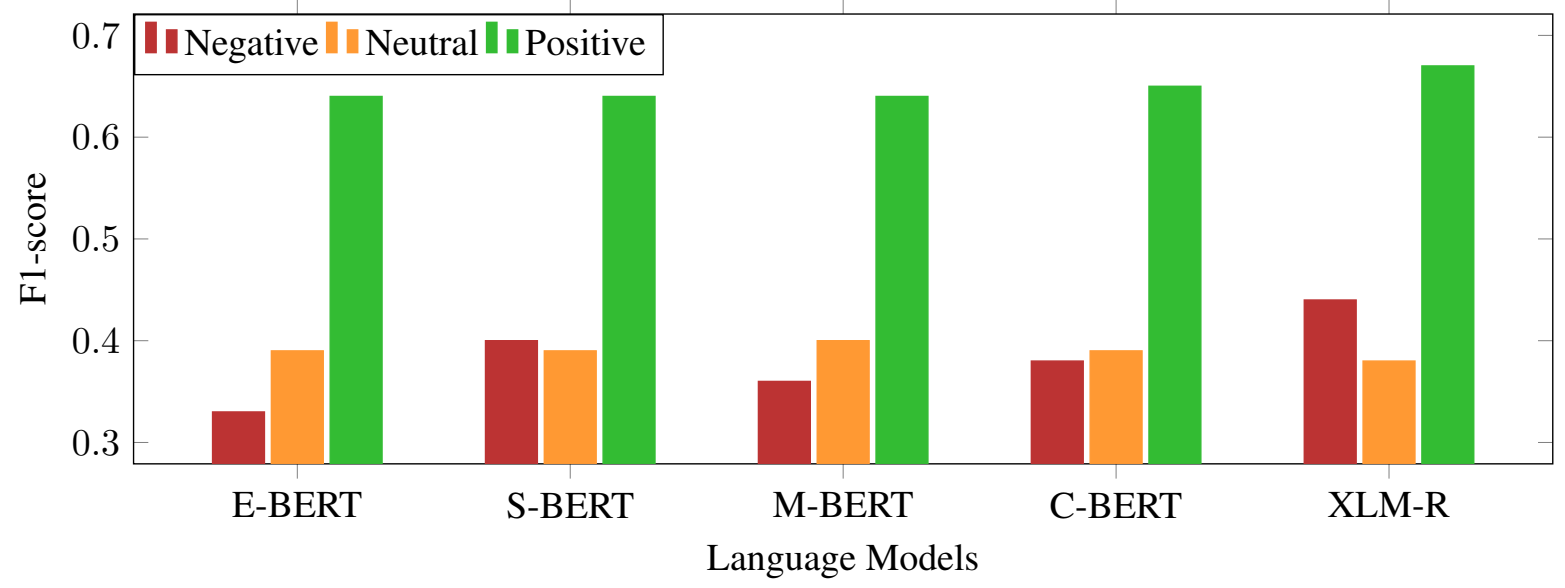

Figure 2: F1-scores for each sentiment label on validation data.

\section{Discussion}

To better understand our results, we performed some error analysis. Since our data consisted of tweets with both English and Spanish words, we expected the multilingual models to perform better than their monolingual counterparts. However, we did not see this pattern in our results. Multilingual BERT model performed better than the English BERT, but worse than the Spanish one. To see in which regard our models differ from each other, we investigated the differences in tokenization, and the effect of language use.

\subsection{Tokenization}

Firstly, we decided to look at the differences between the tokenizers. The pre-trained models in our selection, use either WordPiece (WP) (Wu et al., 2016) or SentencePiece (SP) (Kudo and Richardson, 2018) tokenization, where words are split into substrings (and possibly morphemes). Since Spanish has a more complex morphology system than English (Ramirez et al., 2010), the English tokenizer had great difficulty recognizing the correct morphemes. Based on this observation, we hypothesized that fewer wordpieces corresponded to a more accurate tokenization.

We therefore came up with an evaluation metric: the number of non-first tokens. Firstly, we tokenized the training data using NLTK word_tokenize, which is a word-level tokenizer. Secondly, we tokenized the same data using four different BERT tokenizers. We then subtracted the number of tokens for every tokenizer from the number of words, to calculate the amount of non-first tokens. The results of this evaluation can be seen in Table 2 . 


\begin{tabular}{llcc}
\hline & Tokenizer & Vocabulary size & Non-first tokens \\
\hline 1 & English BERT-base & 30k (WP) & 205,905 \\
2 & Spanish BERT-base & $31 \mathrm{k}(\mathrm{SP})$ & $\mathbf{1 2 7 , 3 2 3}$ \\
3 & Multilingual BERT-base & $110 \mathrm{k}(\mathrm{WP})$ & 152,877 \\
4 & XLM-RoBERTa large & $250 \mathrm{k}(\mathrm{SP})$ & 131,591 \\
\hline
\end{tabular}

Table 2: Number of non-first tokens per tokenizer on training data.

\begin{tabular}{|c|c|}
\hline & Sentence \\
\hline 1 & 'Since', 'I', 'started', 'working', 'ya', 'ni', 'di', '\#\#s', '\#\#f', '\#\#ru', '\#\#to', 'la', 'v', '\#\#ida', 'lo', '\#\#l' \\
\hline 2 & 'Sin', '\#\#ce', 'I', 'sta', '\#\#r', '\#\#ted', 'w', '\#\#or', '\#\#k', '\#\#ing', 'ya', 'ni', 'disfru', '\#\#to', 'la', 'vida', 'lo', '\#\#l' \\
\hline 3 & 'Since', 'I', 'started', 'working', 'ya', 'ni', 'dis', '\#\#fr', '\#\#uto', 'la', 'vida', 'lo', '\#\#l' \\
\hline 4 & '_Since', '_I', '_started', '_working', '_ya', '_ni', '_dis', 'fru', 'to', '_la', '_vida', '_lol' \\
\hline
\end{tabular}

Table 3: Wordpieces produced by each model's tokenizer for an example sentence. Each line corresponds to a model: English BERT (1), Spanish BERT (2), Multilingual BERT (3) and XLM-RoBERTa (4).

Multilingual BERT-base produced considerably fewer tokens than monolingual BERT-base. Again, multilingual XLM-RoBERTa produced fewer non-first tokens than monolingual BERT. These results would indicate that a multilingual tokenizer is better than a monolingual one. However, the monolingual BERT-base Spanish tokenizer breaks this pattern, with the lowest number of non-first tokens.

The increase in number of non-first tokens for the XLM-RoBERTa tokenizer over the multilingual BERT tokenizer might be due to the vocabulary size (i.e., pieces) of the models. XLM-RoBERTa has a vocabulary size of $250 \mathrm{k}$, whereas multilingual BERT uses only a $110 \mathrm{k}$ vocabulary. This shows that tokenization has a clear effect on performance although it is not the only determining variable for the overall accuracy, considering the Spanish case. Table 3 shows an example sentence tokenized by each model's tokenizer.

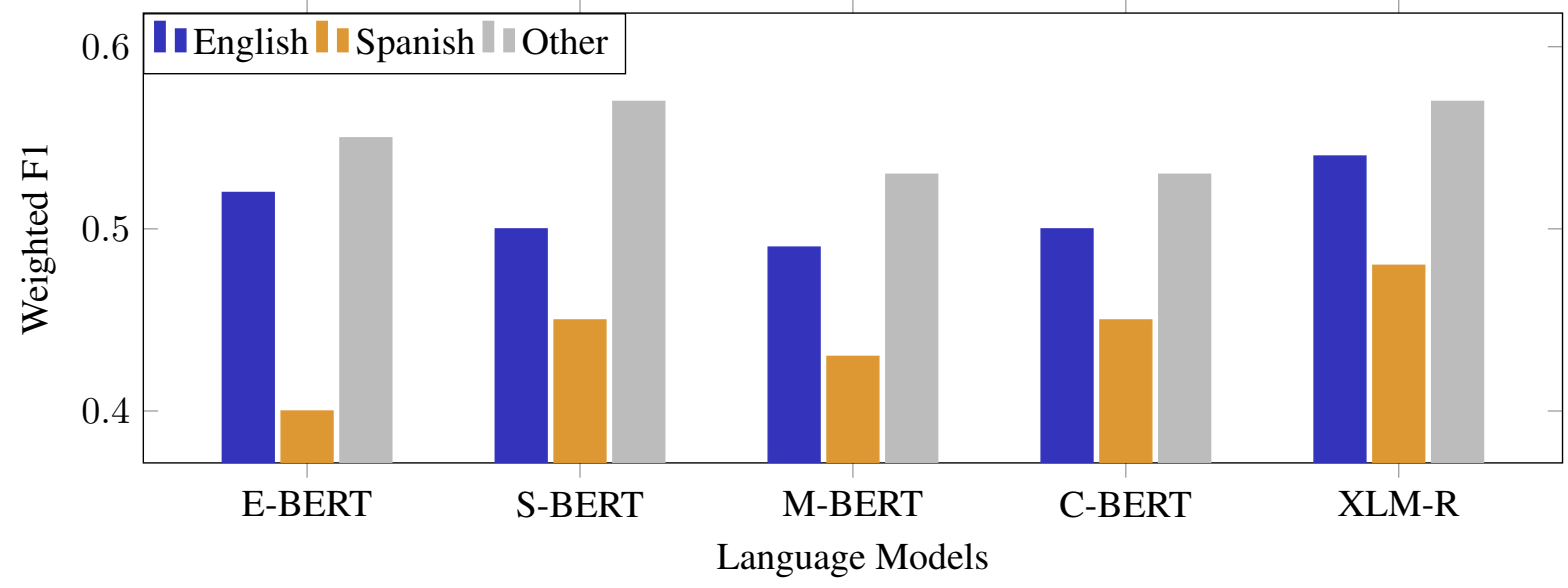

Figure 3: Weighted F1-scores for the Transformer models, separated by language.

\subsection{Language-specific Performance}

As a second evaluation, we looked at the models' performance on sentences with a different ratio of languages (English and Spanish). For this, we split the development dataset into sentences with more Spanish than English words, and vice-versa by using token-level language labels. We also present another group, with miscellaneous labels such as ambiguous and other ${ }^{4}$. We then looked at the predictions, and calculated the weighted F1-score for every language group. The results are shown in Figure 3.

As one would expect, when looking at the monolingual Transformers, we see that the Spanish and

\footnotetext{
${ }^{4}$ We used a threshold of 0.75 for both the English and Spanish splits, and 0.4 for 'Others'.
} 
English BERT models excel at their respective language group. However, while English BERT suffered from very poor performance on predominantly Spanish tweets, its Spanish counterpart had a more balanced performance. Interestingly, although multilingual BERT's performance is on-par with Spanish BERT on the English group, it underperforms on the Spanish group, which would explain why Spanish BERT has a better overall F1-score than its multilingual counterpart.

For the custom model (C-BERT), a two-step fine-tuning strategy to enrich the model with codeswitched domain-specific information (i.e., social media) improved performance over the multilingual BERT. As shown in Figure 3, C-BERT performed better on especially Spanish group of sentences, compared to the it's multilingual base. This indicates that more domain-specific training could increase the quality of a multilingual pre-trained model, considering the task and code-mixing challenge.

Finally, Figure 3 clearly shows why XLM-RoBERTa outperforms all of the other models. It has the best performance on all three groups of sentences, regardless of the language.

\section{Conclusion}

In this paper we presented FiSSA, our approach for sentiment classification on Spanish-English data. We showed that fine-tuning a pre-trained language model is a good alternative to a standard model, especially when the amount of labeled training data is limited. By fine-tuning XLM-RoBERTa, we achieved a weighted F1-score of 0.537 on development data and 0.739 on test data

In the discussion, we evaluated the effect of tokenization and language-specific performance of each model to better understand the overall results. 


\section{References}

Alan Akbik, Tanja Bergmann, and Roland Vollgraf. 2019. Pooled contextualized embeddings for named entity recognition. In NAACL 2019, 2019 Annual Conference of the North American Chapter of the Association for Computational Linguistics, page 724-728.

Kfir Bar and Nachum Dershowitz. 2014. The Tel aviv university system for the code-switching workshop shared task. In Proceedings of the First Workshop on Computational Approaches to Code Switching, pages 139-143, Doha, Qatar, October. Association for Computational Linguistics.

José Cañete, Gabriel Chaperon, Rodrigo Fuentes, and Jorge Pérez. 2020. Spanish pre-trained bert model and evaluation data. In to appear in PMLADC at ICLR 2020.

Alexis Conneau, Kartikay Khandelwal, Naman Goyal, Vishrav Chaudhary, Guillaume Wenzek, Francisco Guzmán, Edouard Grave, Myle Ott, Luke Zettlemoyer, and Veselin Stoyanov. 2019. Unsupervised crosslingual representation learning at scale.

Jacob Devlin, Ming-Wei Chang, Kenton Lee, and Kristina Toutanova. 2018. BERT: pre-training of deep bidirectional transformers for language understanding. CoRR, abs/1810.04805.

Sepp Hochreiter and Jürgen Schmidhuber. 1997. Long short-term memory. Neural Computation, 9(8):17351780.

Taku Kudo and John Richardson. 2018. SentencePiece: A simple and language independent subword tokenizer and detokenizer for neural text processing. In Proceedings of the 2018 Conference on Empirical Methods in Natural Language Processing: System Demonstrations, pages 66-71, Brussels, Belgium, November. Association for Computational Linguistics.

Guillaume Lample and Alexis Conneau. 2019. Cross-lingual language model pretraining. Advances in Neural Information Processing Systems (NeurIPS).

Yinhan Liu, Myle Ott, Naman Goyal, Jingfei Du, Mandar Joshi, Danqi Chen, Omer Levy, Mike Lewis, Luke Zettlemoyer, and Veselin Stoyanov. 2019. Roberta: A robustly optimized bert pretraining approach. arXiv preprint arXiv:1907.11692.

Parth Patwa, Gustavo Aguilar, Sudipta Kar, Suraj Pandey, Srinivas PYKL, Björn Gambäck, Tanmoy Chakraborty, Thamar Solorio, and Amitava Das. 2020. Semeval-2020 task 9: Overview of sentiment analysis of code-mixed tweets. In Proceedings of the 14th International Workshop on Semantic Evaluation (SemEval-2020), Barcelona, Spain, December. Association for Computational Linguistics.

Telmo Pires, Eva Schlinger, and Dan Garrette. 2019. How multilingual is multilingual BERT? In Proceedings of the 57th Annual Meeting of the Association for Computational Linguistics, pages 4996-5001, Florence, Italy, July. Association for Computational Linguistics.

Gloria Ramirez, Xi Chen, Esther Geva, and Heidi Kiefer. 2010. Morphological awareness in spanish-speaking english language learners: Within and cross-language effects on word reading. Reading and Writing, 23(34):337-358.

Thamar Solorio and Yang Liu. 2008. Part-of-speech tagging for english-spanish code-switched text. pages 10511060,01 .

Thamar Solorio, Elizabeth Blair, Suraj Maharjan, Steven Bethard, Mona Diab, Mahmoud Ghoneim, Abdelati Hawwari, Fahad AlGhamdi, Julia Hirschberg, Alison Chang, and Pascale Fung. 2014. Overview for the first shared task on language identification in code-switched data. In Proceedings of the First Workshop on Computational Approaches to Code Switching, pages 62-72, Doha, Qatar, October. Association for Computational Linguistics.

Ashish Vaswani, Noam Shazeer, Niki Parmar, Jakob Uszkoreit, Llion Jones, Aidan N. Gomez, Lukasz Kaiser, and Illia Polosukhin. 2017. Attention is all you need. CoRR, abs/1706.03762.

David Vilares, Miguel A. Alonso, and Carlos Gómez-Rodríguez. 2015. Sentiment analysis on monolingual, multilingual and code-switching twitter corpora. In Proceedings of the 6th Workshop on Computational Approaches to Subjectivity, Sentiment and Social Media Analysis, pages 2-8, Lisboa, Portugal, September. Association for Computational Linguistics. 
David Vilares, Miguel A. Alonso, and Carlos Gómez-Rodríguez. 2016. EN-ES-CS: An English-Spanish codeswitching twitter corpus for multilingual sentiment analysis. In Proceedings of the Tenth International Conference on Language Resources and Evaluation (LREC'16), pages 4149-4153, Portorož, Slovenia, May. European Language Resources Association (ELRA).

Thomas Wolf, Lysandre Debut, Victor Sanh, Julien Chaumond, Clement Delangue, Anthony Moi, Pierric Cistac, Tim Rault, R'emi Louf, Morgan Funtowicz, and Jamie Brew. 2019. Huggingface's transformers: State-of-theart natural language processing. ArXiv, abs/1910.03771.

Yonghui Wu, Mike Schuster, Zhifeng Chen, Quoc V Le, Mohammad Norouzi, Wolfgang Macherey, Maxim Krikun, Yuan Cao, Qin Gao, Klaus Macherey, et al. 2016. Google's neural machine translation system: Bridging the gap between human and machine translation. arXiv preprint arXiv:1609.08144.

Zhilin Yang, Zihang Dai, Yiming Yang, Jaime Carbonell, Ruslan Salakhutdinov, and Quoc V. Le. 2019. Xlnet: Generalized autoregressive pretraining for language understanding. cite arxiv:1906.08237Comment: Pretrained models and code are available at https://github.com/zihangdai/xlnet. 